\title{
Genetic associations between Transcription Factor 7 Like 2 rs7903146 polymorphism and type 2 diabetes mellitus: a meta-analysis of 115,809 subjects
}

\author{
Liying Lou*, Jingjing Wang and Jing Wang
}

\begin{abstract}
Background: Some genetic association studies tried to investigate potential associations of Transcription Factor 7 Like 2 (TCF7L2) rs7903146 polymorphism with type 2 diabetes mellitus (T2DM). However, the results of these studies were not consistent. Thus, we performed the present meta-analysis to explore associations between TCF7L2 rs7903146 polymorphism and T2DM in a larger pooled population.
\end{abstract}

Methods: Systematic literature research of PubMed, Web of Science and Embase was performed to identify eligible studies for pooled analyses. $I^{2}$ statistics were employed to assess between-study heterogeneities. If $I^{2}$ was greater than $50 \%$, random-effect models (REMs) would be used to pool the data. Otherwise, fixed-effect models (FEMs) would be applied for synthetic analyses.

Results: Totally 68 studies with 115,809 subjects were included for analyses. The pooled analyses showed that TCF7L2 rs7903146 (dominant model: $p<0.0001$; recessive model: $p<0.0001$; over-dominant model: $p<0.0001$; allele model: $p<0.0001)$ polymorphism was significantly associated with susceptibility to T2DM in overall population. Further subgroup analyses revealed similar significant findings in both Asians and Caucasians.

Conclusions: In conclusion, our findings supported that TCF7L2 rs 7903146 polymorphism could be used to identify individuals at high risk of developing T2DM in Asians and Caucasians.

Keywords: Transcription Factor 7 Like 2 (TCF7L2), rs7903146 polymorphism, Type 2 diabetes mellitus (T2DM), Metaanalysis

\section{Background}

Type 2 diabetes mellitus (T2DM), characterized by chronic hyperglycemia caused by insufficient responses to insulin, is the most prevalent type of metabolic disorder, and it is estimated that over 344 million people are currently affected by this disease worldwide $[1,2]$. So far, the exact pathogenesis of T2DM is still not fully understood. However, past genome-wide association studies already identified over 100 genetic loci that were significantly associated with an increased susceptibility to

*Correspondence: liyinglou69@163.com

Department of Endocrinology, Shengzhou People's Hospital, No. 666 Dangui Road of Sanjiang Street, Shaoxing 312400, Zhejiang, China
T2DM, which supported that inherit factors were crucial for its occurrence and development $[3,4]$.

Transcription Factor 7 Like 2 (TCF7L2) gene encodes $\mathrm{T}$ cell transcription factor 4 , a transcription factor of the Wnt/ $\beta$-catenin signaling pathway that is vital for embryogenesis of the pancreas islet and regulation of blood glucose [5, 6]. Recently, some genome-wide association studies found that TCF7L2 rs7903146 polymorphism could significantly affect individual susceptibility to T2DM in certain populations [7, 8]. Since then, many genetic association studies were performed in diverse populations to estimate potential associations between TCF7L2 rs7903146 polymorphism and T2DM, with inconsistent results. In 2018, Ding et al. [9] already 
performed a meta-analysis to assess association between TCF7L2 rs7903146 polymorphism and T2DM, but only 28 studies were included by the authors and many eligible studies were missed. Therefore, we conducted an updated meta-analysis of all relevant studies published before May 2019 to more comprehensively analyze the effects of TCF7L2 rs7903146 polymorphism on individual susceptibility to T2DM in a larger pooled population.

\section{Methods}

The current meta-analysis was reported according to the Preferred Reporting Items for Systematic Reviews and Meta-analyses (PRISMA) statement [10].

\section{Literature search and inclusion criteria}

Potentially relevant articles were searched in PubMed, Medline and Web of Science using the following key words: "TCF7L2", "Transcription Factor 7 Like 2", "polymorphism", "variant", "mutation", "SNP", "genotype", "allele", "type 2 diabetes", "type II diabetes" and "T2DM". The initial literature search was performed in January 2019 and the latest update was finished in May 2019. Moreover, we also screened the references of all retrieved articles to identify other potential relevant studies.

Included studies must meet all the following criteria: (1) genetic association studies on associations between TCF7L2 rs7903146 polymorphism and T2DM in human beings; (2) provide genotypic/allelic frequency of TCF7L2 rs7903146 polymorphism in cases and controls; (3) full text in English available. For duplicate reports, only the most complete one was included. Studies were excluded if one of the following criteria was fulfilled: (1) not about TCF7L2 rs7903146 polymorphism and T2DM; (2) studies that were not performed in human beings; (3) case reports or case series; (4) reviews, comments and conference presentations.

\section{Data extraction and quality assessment}

The following data were extracted from included studies: (1) Last name of first author; (2) Year of publication; (3) Country where the study was conducted and ethnicity of study participants; (4) type of disease; (5) the number of cases and controls; and (6) genotypic/allelic distributions of TCF7L2 rs7903146 polymorphism in cases and controls. The probability value ( $p$ value) of Hardy-Weinberg equilibrium (HWE) was also calculated. When necessary, we wrote to the corresponding authors for extra information. We used the Newcastle-Ottawa scale (NOS) to assess the quality of eligible studies [11]. This scale has a score range of zero to nine, and studies with a score of more than seven were thought to be of high quality. Data extraction and quality assessment were performed by two independent reviewers. Any disagreement between two reviewers was solved by discussion until a consensus was reached.

\section{Statistical analyses}

We used Review Manager Version 5.3.3 (The Cochrane Collaboration, Software Update) to conduct statistical analyses. We calculated odds ratios (ORs) and 95\% confidence intervals (CIs) to estimate strength of associations between TCF7L2 rs7903146 polymorphism and T2DM in dominant, recessive, over-dominant and allele models. Statistical significances of pooled analyses were determined by the $\mathrm{Z}$ test, with a $p$ value of 0.05 or less was defined as statistically significant. $\mathrm{I}^{2}$ statistics were employed to assess between-study heterogeneities. If $\mathrm{I}^{2}$ was greater than $50 \%$, random-effect models (REMs) would be used to pool the data on account of significant heterogeneities. Otherwise, fixed-effect models (FEMs) would be used for synthetic analyses. Subgroup analyses by ethnicity of participants were subsequently performed to evaluate effects of ethnic background on investigated genetic associations. Sensitivity analyses were carried out to test the stability of pooled results by omitting one study each time and re-perform analyses based on the results of the remaining studies. Publication biases were evaluated with funnel plots.

\section{Results}

\section{Characteristics of included studies}

The initial literature search found 946 potential relevant articles. After exclusion of irrelevant and duplicate articles by reading titles and abstracts, 278 potentially relevant articles were retrieved for eligibility assessment. Another 210 articles were subsequently excluded after reading the full text. Finally, a total of 68 studies that met the inclusion criteria of our meta-analysis were included (Fig. 1). Baseline characteristics of included studies were shown in Table 1.

\section{TCF7L2 rs7903146 polymorphism and T2DM}

The results of overall and subgroup analyses were summarized in Table 2. Totally 68 studies with 115,809 subjects were included for analyses, the pooled analyses showed that TCF7L2 rs7903146 (dominant model: $p<0.0001$, OR $=0.66,95 \%$ CI $0.63-0.70$; recessive model: $p<0.0001, \mathrm{OR}=1.64,95 \%$ CI $1.56-1.73$; over-dominant model: $p<0.0001, \mathrm{OR}=1.27,95 \%$ CI 1.21-1.34; allele model: $p<0.0001$, OR $=0.71,95 \%$ CI $0.68-0.74$ ) polymorphism was significantly associated with susceptibility to T2DM in overall population. Further subgroup analyses revealed similar significant findings in both Asians and Caucasians (Table 2). 


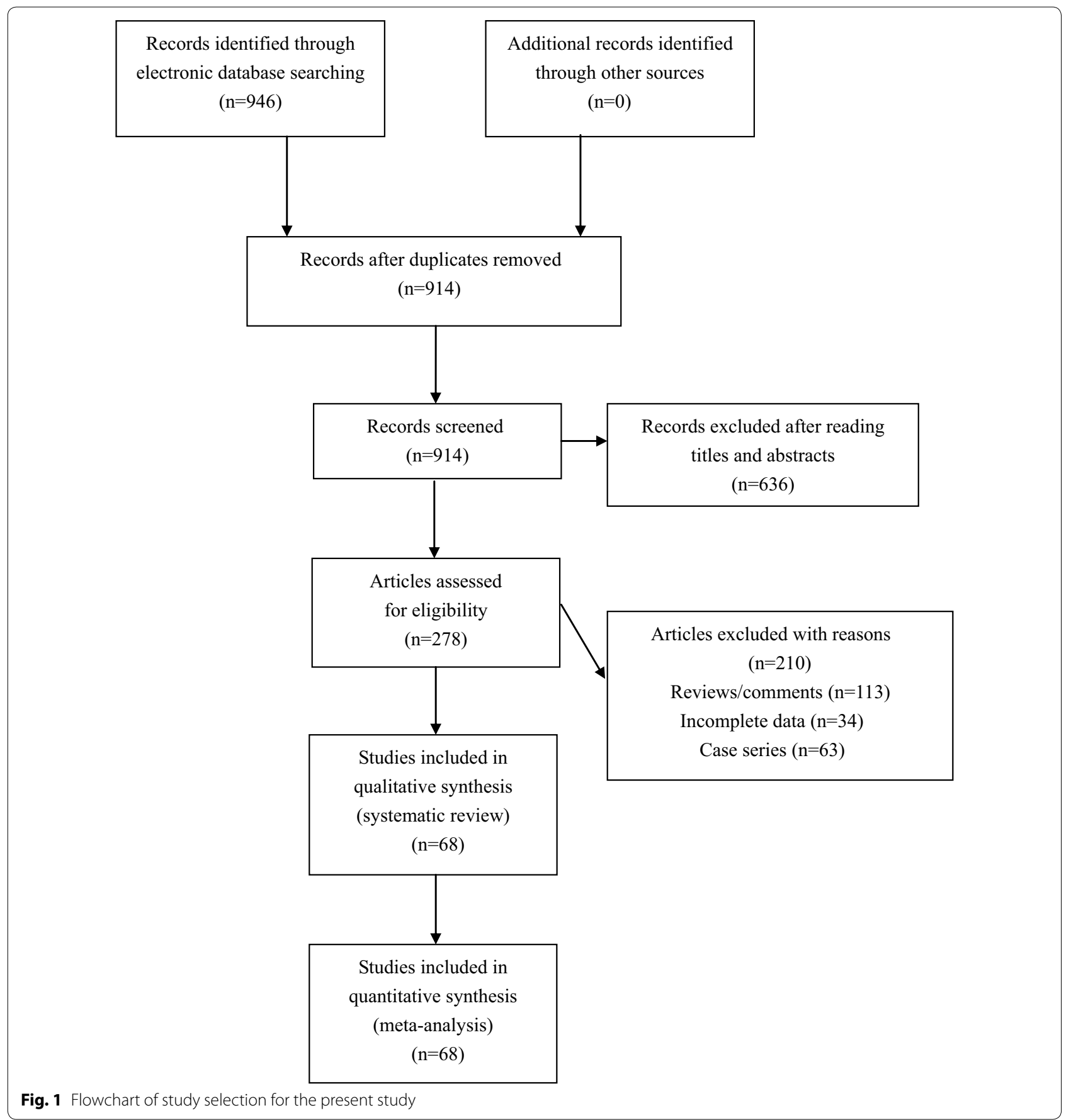

\section{Sensitivity analyses}

We performed sensitivity analyses by deleting one individual study each time to test the effects of individual study on pooled results. No any altered results were observed in overall and subgroup comparisons, which indicated that our findings were statistically robust.

\section{Publication biases}

We used funnel plots to assess publication biases. We did not find obvious asymmetry of funnel plots in any comparisons, which suggested that our findings were unlikely to be impacted by severe publication biases (Additional file 1: Fig. S1). 
Table 1 The characteristics of included studies

\begin{tabular}{|c|c|c|c|c|c|c|c|c|}
\hline \multirow{2}{*}{$\begin{array}{l}\text { First author, } \\
\text { year }\end{array}$} & \multirow[t]{2}{*}{ Country } & \multirow[t]{2}{*}{ Ethnicity } & \multirow[t]{2}{*}{ Type of disease } & \multirow[t]{2}{*}{ Sample size } & \multicolumn{2}{|c|}{ Genotypes (wtwt/wtmt/mtmt) } & \multirow[t]{2}{*}{$p$ value for HWE } & \multirow[t]{2}{*}{ NOS score } \\
\hline & & & & & Cases & Controls & & \\
\hline \multicolumn{9}{|l|}{ rs7903146 C/T } \\
\hline Acharya 2015 & Saudi Arabia & South Asian & $\mathrm{T} 2 \mathrm{DM}$ & $359 / 351$ & $131 / 137 / 91$ & $132 / 143 / 76$ & 0.002 & 8 \\
\hline Al-Sinani 2015 & Oman & South Asian & $\mathrm{T} 2 \mathrm{DM}$ & $992 / 294$ & NA & NA & NA & 7 \\
\hline Anjum 2018 & China & East Asian & $\mathrm{T} 2 \mathrm{DM}$ & $339 / 191$ & $160 / 117 / 62$ & $110 / 56 / 25$ & $<0.001$ & 7 \\
\hline Assmann 2014 & Brazil & Mixed & T2DM & $953 / 535$ & $382 / 415 / 156$ & $261 / 215 / 59$ & 0.147 & 8 \\
\hline Barra 2012 & Brazil & Mixed & T2DM & $113 / 139$ & $49 / 47 / 17$ & $70 / 63 / 6$ & 0.076 & 7 \\
\hline Barros 2014 & Brazil & Mixed & T2DM & 108/109 & $53 / 49 / 6$ & $58 / 40 / 11$ & 0.304 & 7 \\
\hline Beloso 2018 & Uruguay & Mixed & T2DM & $177 / 133$ & $84 / 66 / 27$ & $71 / 47 / 15$ & 0.104 & 7 \\
\hline Bielicki 2019 & Poland & Caucasian & $\mathrm{T} 2 \mathrm{DM}$ & $121 / 479$ & $69 / 45 / 7$ & 285/172/22 & 0.539 & 7 \\
\hline Bodhini 2007 & India & South Asian & $\mathrm{T} 2 \mathrm{DM}$ & $1031 / 1038$ & $462 / 455 / 114$ & $555 / 391 / 92$ & 0.055 & 8 \\
\hline Cai 2019 & China & East Asian & T2DM & $296 / 446$ & 197/83/16 & 287/147/12 & 0.180 & 8 \\
\hline Cauchi 2006 & France & Caucasian & T2DM & $2367 / 2499$ & 787/1149/431 & $1208 / 1060 / 231$ & 0.944 & 8 \\
\hline Chandak 2007 & India & South Asian & $\mathrm{T} 2 \mathrm{DM}$ & $955 / 399$ & $391 / 423 / 141$ & 205/160/34 & 0.726 & 8 \\
\hline Chang 2007 & Taiwan & East Asian & T2DM & $760 / 760$ & NA & NA & NA & 7 \\
\hline $\begin{array}{l}\text { Chidambaram } \\
2016\end{array}$ & India & South Asian & $\mathrm{T} 2 \mathrm{DM}$ & $877 / 838$ & NA & NA & NA & 7 \\
\hline Corella 2016 & Spain & Caucasian & $\mathrm{T} 2 \mathrm{DM}$ & $3411 / 3607$ & $1158 / 1680 / 573$ & $1612 / 1569 / 426$ & 0.140 & 8 \\
\hline Dahlgren 2017 & Sweden & Caucasian & T2DM & $168 / 885$ & $67 / 83 / 18$ & $496 / 327 / 62$ & 0.421 & 8 \\
\hline Danquah 2013 & Germany & Caucasian & T2DM & $674 / 375$ & 273/323/78 & $182 / 165 / 28$ & 0.257 & 7 \\
\hline De Silva 2007 & UK & Caucasian & T2DM & $601 / 2099$ & 211/299/91 & 1032/887/180 & 0.586 & 7 \\
\hline El-Lebedy 2016 & Egypt & Caucasian & T2DM & $180 / 210$ & $48 / 126 / 6$ & $112 / 95 / 3$ & $<0.001$ & 8 \\
\hline $\begin{array}{l}\text { Erkoç Kaya } \\
2017\end{array}$ & Turkey & Caucasian & $\mathrm{T} 2 \mathrm{DM}$ & $171 / 120$ & $58 / 95 / 18$ & $57 / 47 / 16$ & 0.215 & 7 \\
\hline Ezzidi 2009 & Tunisia & Caucasian & $\mathrm{T} 2 \mathrm{DM}$ & $863 / 511$ & 250/396/217 & 181/235/95 & 0.227 & 8 \\
\hline Groves 2006 & UK & Caucasian & T2DM & $2001 / 2476$ & $771 / 960 / 270$ & $1175 / 1084 / 217$ & 0.139 & 8 \\
\hline $\begin{array}{l}\text { Guewo-Fokeng } \\
2015\end{array}$ & Cameroon & African & $\mathrm{T} 2 \mathrm{DM}$ & $74 / 74$ & $37 / 30 / 7$ & $37 / 37 / 0$ & 0.004 & 7 \\
\hline Gupta 2010 & India & South Asian & T2DM & 195/161 & $55 / 96 / 44$ & $62 / 78 / 21$ & 0.647 & 8 \\
\hline Hayashi 2007 & Japan & East Asian & $\mathrm{T} 2 \mathrm{DM}$ & $1619 / 1069$ & $1450 / 165 / 4$ & $980 / 85 / 2$ & 0.146 & 8 \\
\hline Horikoshi 2007 & Japan & East Asian & T2DM & $1174 / 823$ & $1051 / 119 / 4$ & $770 / 51 / 2$ & 0.243 & 8 \\
\hline Hsiao 2017 & Taiwan & East Asian & T2DM & $562 / 986$ & $497 / 62 / 3$ & $933 / 52 / 1$ & 0.755 & 7 \\
\hline $\begin{array}{l}\text { Humphries } \\
2016\end{array}$ & UK & Caucasian & $\mathrm{T} 2 \mathrm{DM}$ & $1459 / 2493$ & $601 / 665 / 193$ & 1295/1001/197 & 0.854 & 7 \\
\hline $\begin{array}{l}\text { Humphries } \\
2016\end{array}$ & UK & South Asian & $\mathrm{T} 2 \mathrm{DM}$ & $837 / 300$ & $366 / 375 / 96$ & 163/111/26 & 0.260 & 7 \\
\hline $\begin{array}{l}\text { Humphries } \\
2016\end{array}$ & UK & African & $\mathrm{T} 2 \mathrm{DM}$ & $307 / 311$ & $141 / 136 / 30$ & $161 / 124 / 26$ & 0.759 & 7 \\
\hline Hussain 2014 & India & South Asian & T2DM & $123 / 82$ & $45 / 63 / 15$ & $43 / 35 / 4$ & 0.350 & 7 \\
\hline Isakova 2019 & Kyrgyzstan & Caucasian & $\mathrm{T} 2 \mathrm{DM}$ & $114 / 109$ & $91 / 20 / 3$ & $89 / 16 / 4$ & 0.009 & 8 \\
\hline Jia 2016 & China & East Asian & $\mathrm{T} 2 \mathrm{DM}$ & $248 / 267$ & $125 / 73 / 50$ & $165 / 74 / 28$ & $<0.001$ & 8 \\
\hline Kalantari 2019 & Iran & South Asian & T2DM & $530 / 420$ & $155 / 241 / 134$ & 187/173/60 & 0.056 & 7 \\
\hline Katsoulis 2018 & Greece & Caucasian & T2DM & $148 / 80$ & $30 / 104 / 14$ & $54 / 23 / 3$ & 0.779 & 7 \\
\hline Khan 2015 & India & South Asian & T2DM & $42 / 98$ & 13/18/11 & $57 / 33 / 8$ & 0.312 & 7 \\
\hline Khan 2015 & India & South Asian & T2DM & $250 / 250$ & $92 / 120 / 38$ & $144 / 87 / 19$ & 0.255 & 7 \\
\hline Kimber 2007 & UK & Caucasian & T2DM & $3225 / 3291$ & $1405 / 1459 / 361$ & $1714 / 1329 / 248$ & 0.663 & 8 \\
\hline Kong 2015 & China & East Asian & T2DM & $5169 / 4560$ & NA & NA & NA & 7 \\
\hline Kunika 2008 & Japan & East Asian & $\mathrm{T} 2 \mathrm{DM}$ & $1422 / 1423$ & $1246 / 171 / 5$ & 1309/111/3 & 0.689 & 8 \\
\hline $\begin{array}{l}\text { Löfvenborg } \\
2019\end{array}$ & Sweden & Caucasian & T2DM & $1242 / 1530$ & NA & NA & NA & 7 \\
\hline
\end{tabular}


Table 1 (continued)

\begin{tabular}{|c|c|c|c|c|c|c|c|c|}
\hline \multirow{2}{*}{$\begin{array}{l}\text { First author, } \\
\text { year }\end{array}$} & \multirow[t]{2}{*}{ Country } & \multirow[t]{2}{*}{ Ethnicity } & \multirow[t]{2}{*}{ Type of disease } & \multirow[t]{2}{*}{ Sample size } & \multicolumn{2}{|c|}{ Genotypes (wtwt/wtmt/mtmt) } & \multirow[t]{2}{*}{$p$ value for HWE } & \multirow[t]{2}{*}{ NOS score } \\
\hline & & & & & Cases & Controls & & \\
\hline $\begin{array}{l}\text { Marquezine } \\
2008\end{array}$ & Brazil & Mixed & $\mathrm{T} 2 \mathrm{DM}$ & $285 / 1681$ & $83 / 160 / 42$ & $684 / 833 / 164$ & $<0.001$ & 8 \\
\hline Mayans 2007 & Sweden & Caucasian & $\mathrm{T} 2 \mathrm{DM}$ & $824 / 820$ & $452 / 318 / 54$ & $532 / 253 / 35$ & 0.481 & 8 \\
\hline $\begin{array}{l}\text { Miranda-Lora } \\
2017\end{array}$ & Mexico & Mixed & T2DM & $156 / 212$ & $115 / 38 / 3$ & $157 / 51 / 4$ & 0.952 & 8 \\
\hline Miyake 2008 & Japan & East Asian & T2DM & 2154/1834 & $1921 / 228 / 5$ & $1696 / 137 / 1$ & 0.295 & 8 \\
\hline Moran 2015 & Venezuela & African & $\mathrm{T} 2 \mathrm{DM}$ & $70 / 73$ & $26 / 35 / 9$ & $46 / 22 / 5$ & 0.307 & 8 \\
\hline Musavi 2015 & Iran & South Asian & $\mathrm{T} 2 \mathrm{DM}$ & $70 / 100$ & 19/36/15 & $45 / 48 / 7$ & 0.222 & 7 \\
\hline $\begin{array}{l}\text { Ouhaibi-Djel- } \\
\text { louli } 2014\end{array}$ & Algeria & African & T2DM & $76 / 644$ & $16 / 41 / 19$ & 228/287/129 & 0.027 & 8 \\
\hline Palizban 2017 & Iran & South Asian & T2DM & $204 / 80$ & $60 / 95 / 49$ & $32 / 41 / 7$ & 0.224 & 8 \\
\hline Palmer 2011 & USA & Mixed & $\mathrm{T} 2 \mathrm{DM}$ & $982 / 1039$ & NA & NA & NA & 7 \\
\hline $\begin{array}{l}\text { Papandreou } \\
2019\end{array}$ & Spain & Caucasian & T2DM & $869 / 244$ & $382 / 383 / 104$ & $106 / 103 / 35$ & 0.225 & 8 \\
\hline $\begin{array}{l}\text { Plengvidhya } \\
2018\end{array}$ & Thailand & East Asian & T2DM & $500 / 500$ & $429 / 67 / 4$ & $456 / 44 / 0$ & 0.303 & 8 \\
\hline $\begin{array}{l}\text { Pourahmadi } \\
2015\end{array}$ & Iran & South Asian & $\mathrm{T} 2 \mathrm{DM}$ & $200 / 200$ & 109/68/23 & $126 / 59 / 15$ & 0.037 & 8 \\
\hline Rees 2008 & UK & South Asian & $\mathrm{T} 2 \mathrm{DM}$ & $828 / 432$ & $352 / 360 / 116$ & $222 / 166 / 44$ & 0.122 & 8 \\
\hline $\begin{array}{l}\text { Reyes-López } \\
2019\end{array}$ & Mexico & Mixed & T2DM & $23 / 83$ & $14 / 6 / 3$ & $59 / 24 / 0$ & 0.124 & 7 \\
\hline Saadi 2008 & $\begin{array}{l}\text { United Arab } \\
\text { Emirates }\end{array}$ & South Asian & $\mathrm{T} 2 \mathrm{DM}$ & $180 / 188$ & $56 / 103 / 21$ & $71 / 94 / 23$ & 0.339 & 7 \\
\hline Scott 2006 & USA & Mixed & $\mathrm{T} 2 \mathrm{DM}$ & $1151 / 953$ & NA & NA & NA & 7 \\
\hline Tabara 2009 & Japan & East Asian & T2DM & $481 / 398$ & $434 / 45 / 2$ & $372 / 26 / 0$ & 0.501 & 8 \\
\hline Turki 2013 & Tunisia & South Asian & T2DM & $895 / 878$ & $255 / 432 / 208$ & $330 / 414 / 134$ & 0.824 & 7 \\
\hline $\begin{array}{l}\text { Uma Jyothi } \\
2015\end{array}$ & India & South Asian & $\mathrm{T} 2 \mathrm{DM}$ & $758 / 621$ & $341 / 326 / 83$ & $391 / 193 / 37$ & 0.048 & 7 \\
\hline $\begin{array}{l}\text { van Vliet- } \\
\text { Ostaptchouk } \\
2007\end{array}$ & Netherlands & Caucasian & $\mathrm{T} 2 \mathrm{DM}$ & $496 / 907$ & 203/221/72 & $459 / 365 / 83$ & 0.397 & 7 \\
\hline Včelák 2012 & Czech Republic & Caucasian & $\mathrm{T} 2 \mathrm{DM}$ & $347 / 376$ & $148 / 156 / 43$ & $205 / 147 / 24$ & 0.731 & 8 \\
\hline Wang 2013 & China & East Asian & $\mathrm{T} 2 \mathrm{DM}$ & 1842/7777 & $1553 / 283 / 6$ & $6718 / 1032 / 27$ & 0.057 & 8 \\
\hline Wrzosek 2019 & Poland & Caucasian & $\mathrm{T} 2 \mathrm{DM}$ & $129 / 345$ & $67 / 50 / 12$ & 219/113/13 & 0.738 & 8 \\
\hline Yako 2015 & South Africa & African & T2DM & $152 / 328$ & $66 / 74 / 12$ & $184 / 129 / 15$ & 0.199 & 8 \\
\hline Yu 2009 & USA & Mixed & T2DM & $686 / 305$ & $355 / 271 / 60$ & $170 / 111 / 24$ & 0.330 & 8 \\
\hline Zhang 2016 & China & East Asian & $\mathrm{T} 2 \mathrm{DM}$ & $227 / 5284$ & $200 / 24 / 3$ & $4567 / 701 / 16$ & 0.045 & 8 \\
\hline Zheng 2012 & China & East Asian & T2DM & $227 / 152$ & $202 / 24 / 1$ & 139/13/0 & 0.582 & 8 \\
\hline Zhu 2017 & China & East Asian & T2DM & $497 / 782$ & $478 / 19 / 0$ & $740 / 41 / 1$ & 0.584 & 8 \\
\hline Zhuang 2018 & China & East Asian & T2DM & $90 / 96$ & $54 / 26 / 10$ & $69 / 24 / 3$ & 0.611 & 7 \\
\hline
\end{tabular}

T2DM type 2 diabetes mellitus, wt Wild type, $m t$ mutant type, HWE Hardy-Weinberg equilibrium, NOS Newcastle-ottawa scale, NA not available

\section{Discussion}

Despite prominent advancements achieved in drug therapy over the last few decades, T2DM and its associated vascular complications are still leading causes of death and disability around the world $[12,13]$. The exact cause of T2DM is still largely unclear in spite of extensive explorations. However, the obvious familial aggregation tendency of T2DM indicated that genetic factors played significant parts in its pathogenesis [14]. Thus, identify genetic biomarkers is of particularly importance for an early diagnosis and a better prognosis of T2DM patients.

TCF7L2, a box-containing transcription factor that is vital for blood glucose homeostasis, is considered to act through regulation of proglucagon gene expression in enteroendocrine cells via the Wnt signaling pathway [15], and pre-clinical studies also found that TCF7L2 
Table 2 Results of overall and subgroup analyses

\begin{tabular}{llllll}
\hline Variables & Sample size & $\begin{array}{l}\text { Dominant comparison } \\
\boldsymbol{p} \text { value OR }(\mathbf{9 5 \%} \mathrm{Cl})\end{array}$ & $\begin{array}{l}\text { Recessive comparison } \\
\boldsymbol{p} \text { value OR } \mathbf{9 5 \%} \mathrm{Cl})\end{array}$ & $\begin{array}{l}\text { Over-dominant comparison } \\
\boldsymbol{p} \text { value OR } \mathbf{9 5 \%} \mathrm{Cl})\end{array}$ & $\begin{array}{l}\text { Allele comparison } \\
\boldsymbol{p} \text { value OR (95\% Cl) }\end{array}$ \\
\hline Overall & $51,656 / 64,153$ & $<0.00010 .66(0.63-0.70)$ & $<0.00011 .64(1.56-1.73)$ & $<0.00011 .27(1.21-1.34)$ & $<0.00010 .71(0.68-0.74)$ \\
Caucasian & $19,410 / 23,456$ & $<0.00010 .64(0.58-0.70)$ & $<0.00011 .64(1.54-1.75)$ & $<0.00011 .31(1.21-1.43)$ & $<0.00010 .70(0.65-0.75)$ \\
East Asian & $17,607 / 27,348$ & $<0.00010 .73(0.63-0.83)$ & $<0.00011 .90(1.46-2.46)$ & $0.00061 .28(1.11-1.48)$ & $<0.00010 .74(0.66-0.83)$ \\
South Asian & $9326 / 6730$ & $<0.00010 .63(0.59-0.68)$ & $<0.00011 .65(1.48-1.84)$ & $<0.00011 .24(1.16-1.33)$ & $<0.00010 .65(0.60-0.71)$ \\
\hline
\end{tabular}

OR odds ratio, $\mathrm{Cl}$ confidence interval, NA not available, T2DM type 2 diabetes mellitus

expression is positively associated with insulin gene expression in human islets [16]. Considering the vital role of TCF7L2 in regulating blood glucose, many genetic association studies were performed in diverse populations to investigate whether functional TCF7L2 polymorphisms could impact individual susceptibility to T2DM. To our knowledge, this is to date the most comprehensive meta-analysis on association between TCF7L2 rs7903146 polymorphism and T2DM, and our pooled analyses suggested that TCF7L2 rs7903146 polymorphism was significantly associated with T2DM in both Asians and Caucasians. The stabilities of synthetic results were evaluated by sensitivity analyses, and no alterations of results were observed in any comparisons, which suggested that our findings were statistically robust. Significant heterogeneities were detected for dominant and allele comparisons, thus pooled analyses for these two genetic models were performed with REMs. But in further subgroup analyses, an obvious reduction tendency of heterogeneity was found in both Asians and Caucasians, which suggested that differences in ethnic background could largely explain observed heterogeneities between studies. Nevertheless, it is worth noting that the obvious heterogeneities existed among included studies indicated that the distribution of TCF7L2 rs7903146 polymorphism varies greatly from population to population. Therefore, the genetic association between TCF7L2 rs7903146 polymorphism and T2DM may be ethnic-specific, and we should not generalize the subgroup analyses results to a broader population.

There are several points that need to be pointed out about the current study. First, the exact underlying molecular mechanisms of our positive findings remains to be explored, but we speculated that TCF7L2 rs7903146 polymorphism may lead to alternations in gene expression or changes in protein structure, which may subsequently affect biological functions of TCF7L2, impact insulin secretion or decrease sensitivity to insulin, and ultimately affect individual susceptibility to T2DM. Second, the pathogenic mechanism of T2DM is extremely complex, and hence despite our positive findings, it is unlikely that a single gene polymorphism could significantly contribute to its development, and thus we strongly recommend further studies to perform haplotype analyses and explore potential gene-gene interactions $[17,18]$. Third, to more precisely measure the effects of certain genetic factors on disease occurrence and development, gene-environmental interactions should also be considered. However, since included studies only focused on the effects of TCF7L2 rs7903146 polymorphism on individual susceptibility to T2DM, such analyses were not applicable in the current meta-analysis. But to better elucidate the underlying pathogenesis mechanisms of T2DM, future studies should try to investigate the interaction of TCF7L2 gene polymorphisms with potential pathogenic environmental factors such as unhealthy diets or lack of exercise [19]. Our meta-analysis certainly has some limitations. Firstly, although methodology qualities of included studies were generally good, it should be noted that we did not have access to genotypic distributions of investigated polymorphisms according to base characteristics of study subjects. Therefore, our results were derived from unadjusted estimations, and failure to conduct further adjusted analyses for baseline characteristics of participants such as age, gender and comorbidity conditions may influence the veracity of our findings [20, 21]. Secondly, significant heterogeneities were detected in certain subgroup comparisons, which indicated that the inconsistent results of included studies could not be fully explained by differences in ethnic background, and other unmeasured characteristics of participants may also partially attribute to between-study heterogeneities [22]. Thirdly, since only published articles were eligible for analyses, although funnel plots revealed no obvious publication biases, we still could not rule out the possibility of potential publication biases [23]. Taken these limitations into consideration, the results of the current study should be interpreted with caution.

\section{Conclusions}

In conclusion, our findings indicated that TCF7L2 rs7903146 polymorphism was significantly associated with altered susceptibility to T2DM in both Asians and Caucasians. These results supported that this 
polymorphism may be used to identify individuals at high risk of developing T2DM in Asians and Caucasians. Further well-designed studies need to explore possible associations between other TCF7L2 gene polymorphisms and T2DM.

\section{Additional file}

Additional file 1: Figure S1. Funnel plots.

\section{Abbreviations}

TCF7L2: Transcription Factor 7 Like 2: T2DM: type 2 diabetes mellitus; HWE: Hardy-Weinberg equilibrium; NOS: Newcastle-Ottawa scale; REM: randomeffect model; FEM: fixed-effect model.

\section{Acknowledgements}

None.

\section{Authors' contributions}

LL conceived of the study, participated in its design. $L L$ and JW conducted the systematic literature review. JW performed data analyses. LL drafted the manuscript. All authors read and approved the final manuscript.

\section{Funding}

None.

\section{Availability of data and materials}

The current study was based on results of relevant published studies.

\section{Ethics approval and consent to participate}

Not applicable.

\section{Consent for publication}

Not applicable.

\section{Competing interests}

The authors declare that they have no competing interests.

Received: 5 May 2019 Accepted: 24 June 2019

Published online: 05 July 2019

\section{References}

1. Tao Z, Shi A, Zhao J. Epidemiological perspectives of diabetes. Cell Biochem Biophys. 2015;73:181-5.

2. American Diabetes Association. Diagnosis and classification of diabetes mellitus. Diabetes Care. 2014;37(Suppl 1):S81-90.

3. Papazafiropoulou AK, Papanas N, Melidonis A, Maltezos E. Family history of type 2 diabetes: does having a diabetic parent increase the risk? Curr Diabetes Rev. 2017;13:19-25.

4. Gaulton KJ. Mechanisms of type 2 diabetes risk loci. Curr Diab Rep. 2017;17:72.

5. Mondal AK, Das SK, Baldini G, Chu WS, Sharma NK, Hackney OG, Zhao J, Grant SF, Elbein SC. Genotype and tissue-specific effects on alternative splicing of the transcription factor 7-like 2 gene in humans. J Clin Endocrinol Metab. 2010;95:1450-7.
6. Ferreira MC, da Silva MER, Fukui RT, Arruda-Marques MDC, Dos Santos RF. TCF7L2 correlation in both insulin secretion and postprandial insulin sensitivity. Diabetol Metab Syndr. 2018;10:37.

7. Humphries SE, Gable D, Cooper JA, Ireland H, Stephens JW, Hurel SJ, Li KW, Palmen J, Miller MA, Cappuccio FP, Elkeles R, Godsland I, Miller GJ, Talmud PJ. Common variants in the TCF7L2 gene and predisposition to type 2 diabetes in UK European Whites, Indian Asians and Afro-Caribbean men and women. J Mol Med. 2006;84:1005-14.

8. Kimber $\mathrm{CH}$, Doney AS, Pearson ER, McCarthy MI, Hattersley AT, Leese GP, Morris AD, Palmer CN. TCF7L2 in the Go-DARTS study: evidence for a gene dose effect on both diabetes susceptibility and control of glucose levels. Diabetologia. 2007;50:1186-91.

9. Ding W, Xu L, Zhang L, Han Z, Jiang Q, Wang Z, Jin S. Meta-analysis of association between TCF7L2 polymorphism rs7903146 and type 2 diabetes mellitus. BMC Med Genet. 2018;19:38.

10. Moher D, Liberati A, Tetzlaff J, Altman DG, PRISMA Group. Preferred reporting items for systematic reviews and meta-analyses: the PRISMA statement. Ann Intern Med. 2009;151:264-9.

11. Stang A. Critical evaluation of the Newcastle-Ottawa scale for the assessment of the quality of nonrandomized studies in meta-analyses. Eur J Epidemiol. 2010;25:603-5.

12. Nathan DM. Diabetes: advances in diagnosis and treatment. JAMA. 2015;314:1052-62.

13. GBD 2013 Mortality and Causes of Death Collaborators. Global, regional, and national age-sex specific all-cause and cause-specific mortality for 240 causes of death, 1990-2013: a systematic analysis for the Global Burden of Disease Study 2013. Lancet. 2015:385:117-71.

14. Antosik K, Borowiec M. Genetic factors of diabetes. Arch Immunol Ther Exp (Warsz). 2016;64(Suppl 1):157-60.

15. Li R, Ou J, Li L, Yang Y, Zhao J, Wu R. The Wnt signaling pathway effector TCF7L2 mediates olanzapine-induced weight gain and insulin resistance. Front Pharmacol. 2018;9:379.

16. Osmark P, Hansson O, Jonsson AM, Ronn T, Groop L, Renstrom E. Unique splicing pattern of the tcf7l2 gene in human pancreatic islets. Diabetologia. 2009;52:850-4.

17. Szabo M, Máté B, Csép K, Benedek T. Genetic approaches to the study of gene variants and their impact on the pathophysiology of type 2 diabetes. Biochem Genet. 2018;56:22-55.

18. Steen KV. Travelling the world of gene-gene interactions. Brief Bioinform. 2012:13:1-19

19. Nishi A, Milner DA Jr, Giovannucci EL, Nishihara R, Tan AS, Kawachi I, et al. Integration of molecular pathology, epidemiology and social science for global precision medicine. Expert Rev Mol Diagn. 2016;16:11-23.

20. Xie X, Shi X, Liu M. The roles of TLR gene polymorphisms in atherosclerosis: a systematic review and meta-analysis of 35,317 subjects. Scand J Immunol. 2017:86:50-8.

21. Shi $X$, Xie $X$, Jia Y, Li S. Associations of insulin receptor and insulin receptor substrates genetic polymorphisms with polycystic ovary syndrome: a systematic review and meta-analysis. J Obstet Gynaecol Res. 2016;42:844-54.

22. Ma R, Yang H, Li J, Yang X, Chen X, Hu Y, Wang Z, Xue L, Zhou W. Association of HNF4a gene polymorphisms with susceptibility to type 2 diabetes. Mol Med Rep. 2016:13:2241-6.

23. Su S, Zhang C, Zhang F, Li H, Yang X, Tang X. The association between leptin receptor gene polymorphisms and type 2 diabetes mellitus: a systematic review and meta-analysis. Diabetes Res Clin Pract. 2016;121:49-58.

\section{Publisher's Note}

Springer Nature remains neutral with regard to jurisdictional claims in published maps and institutional affiliations. 